\title{
EFFICIENT CALCULATION OF CRITICAL EIGENVALUE CLUSTERS IN THE SMALL SIGNAL STABILITY ANALYSIS OF LARGE POWER SYSTEMS
}

\author{
George Angelidis \\ Adam Semlyen \\ Department of Electrical and Computer Engineering \\ University of Toronto \\ Toronto, Ontario, Canada M5S 1A4
}

\begin{abstract}
The paper presents a methodology for the calculation of a selected set of eigenvalues, considered critical in the small signal stability analysis of power systems. It analyzes several alternatives for refining a preliminary rough solution obtained by subspace iterations. These alternatives range from constant-matrix iterative refinement to Newton's method. Due to an adaptive solution strategy, the overall algorithm is very robust. Newton's method is much faster than existing approaches. The performance of these methods is demonstrated on several test systems.

Keywords: Power system dynamics, Small signal stability, Selective eigenvalue analysis, Eigenvalue clusters, Subspace iterations, Iterative refinement, Newton's method, Sparse matrices.
\end{abstract}

\section{INTRODUCTION}

The small signal stability analysis of large power systems is a difficult task as the size of the problem prohibits the computation of the complete set of eigenvalues by the conventional $Q R$ algorithm. The reason for this is that the state matrix of the state-space equations that describe the dynamic behavior of the power system is dense. Oscillatory modes have been successfully calculated by the AESOPS program [1] that subsequently was significantly improved [2]. Sparse eigenanalysis techniques that exploit sparsity by not reducing the system equations, have been applied on large power systems [3-6]. Alternatively, several methods have been developed for selective eigenpair calculation $[7-12]$. Since the critical modes are usually the only ones of interest in the small signal stability analysis of a power system, selective eigenanalysis methods seek the eigenvalues and the associated eigenvectors in the vicinity of the imaginary axis of the complex plane.

In this paper, we present a methodology for calculating eigenvalues, and their associated eigenvectors, near a specified shif point on the complex plane. An eigenvalue cluster can be identified either as a whole or sequentially. The method is based on a preliminary solution obtained from Subspace (simultaneous) Iterations. This or similar procedures, such as the Modified Arnoldi method, although very robust, are often very slow. Therefore, we use Subspace Iterations only for the purpose of obtaining satisfactory starting values. The contribution of this paper is the development of Iterative Refinement methods [13] to achieve efficiently a high level of accuracy. The alternatives described are: Iterative Refinement with a constant iteration matrix; with a modified iteration matrix, representing a Quasi-Newton approach; and with the Jacobian as iteration matrix, a true Newton method.

94 SM 556-1 PWRS A paper recommended and approved by the IEEE Power System Engineering Committee of the IEEE Power Engineering Society for presentation at the IEEE/PES 1994 Summer Meeting, San Francisco, CA, July $24-28,1994$. Manuscript subaitted December 22, 1993; made available for printing May 3, 1994 .
In the formulation of the problem we have two types of variables: state variables in the dynamic components (generators and their controls, dynamic loads) and algebraic variables (bus voltages and currents). The method is applied to the combined system of equations, without any reduction, in order to maintain sparsity.

The mathematical derivations in the paper have been kept simple but rigorous without undue formalism. All underlying concepts and traditional procedures are presented and analyzed in widely used texts of linear algebra and matrix computations [13-17].

The algorithms have been tested in systems of different size. Some numerical results, showing the performance of the methods, will be presented and discussed.

\section{PROBLEM FORMULATION}

\section{Network Modeling}

For large scale studies, it is common practice to neglect network dynamics, representing the power system interconnection as a lumped, linear, passive network [18]. This approximation is normally acceptable in small signal dynamic analysis where the modes that correspond to network transients are much faster than the modes we are interested in. Therefore, neglecting network dynamics, the power system network is described by the equation

$$
y=Y u
$$

where $y$ and $u$ are the increments of the nodal current injections and voltages, respectively, around the steady state operating point, and $Y$ the nodal admittance matrix. For a network composed of $n$ nodes, equation (1) is a set of $2 n$ real equations, where $y$ and $u$ contain the real and imaginary parts of the corresponding complex phasor increments. Correspondingly, $Y$ has the sparse structure of the complex nodal admittance matrix, with each complex element replaced by a $2 \times 2$ real submatrix.

Dynamic Components

For any such component $k$ we have the linearized state equations

$$
\begin{aligned}
& \dot{x}_{k}=A_{k} x_{k}+B_{k} u_{k} \\
& y_{k}=C_{k} x_{k}+D_{k} u_{k}
\end{aligned}
$$

where the variables are incremental. These equations should be consistent with the network model. For example, since the network dynamics are neglected, the synchronous machine stator dynamics should also be neglected. We note that in (2), the input $u_{k}$ represents part of the nodal voltages $u$ in (1), and a similar remark applies to the output, the current injections $y$ in (1).

\section{Static Loads}

Most loads are usually represented as static, that is as real and reactive powers, functions of the nodal voltage. It is easy to show that all static loads are described by an equation similar to (1)

$$
y_{l}=Y_{l} u_{l}
$$

where the matrices $Y_{l}$ are of dimension $2 \times 2$. Again, the incremental variables $y_{l}$ and $u_{t}$ in (3) are part of the respective variables in (1). 


\section{Combined Equations}

Equations (1), (2b), and (3) can be combined into a single equation by noting that the current injections at each bus, into the network and into the connected static or dynamic components, must satisfy Kirchhoff's current law. Accordingly, the resultant set of equations for the whole system becomes

$$
\begin{aligned}
& \dot{x}=A x+B u \\
& 0=C x+D u
\end{aligned}
$$

We note that in (4) matrices $A, B$ and $C$ have a block-diagonal structure and $D$ has the size and structure of matrix $Y$, being only modified in its $2 \times 2$ diagonal blocks. in the form

For the purpose of eigenvalue analysis we write equations (4)

$$
\begin{aligned}
\lambda x & =A x+B u \\
0 & =C x+D u
\end{aligned}
$$

These may be reduced to the form

$$
A^{\prime} x=\lambda x
$$

by eliminating the algebraic variables, the voltages $u$. Here

$$
A^{\prime}=A-B D^{-1} C
$$
be solved in cases of moderate size by the $Q R$ algorithm. It has also been used for selective eigenvalue analysis [12]. The drawback of this formulation is that the state matrix $A^{\prime}$ is dense due to the reduction process in (7).

In the following, we will use the reduced formulation (6) only for deriving and presenting the basic ideas. In the actua implementation the sparse formulation (5) is used instead.

\section{METHODOLOGY}

\section{Basic Approach}

Traditionally, small subsets of the eigenvalues and the corresponding eigenvectors of large systems have been calculated by variants, generalizations and extensions of the inverse power method, including Subspace Iterations and Arnoldi algorithms. Recently, however, new approaches have been described in the literature. These include derivatives of Newton's method [4,12]. Their advantage is fast convergence. However, they require good starting values that may require significant computational effort [12] Moreover, they require special safeguards to prevent repeated convergence to the same eigenpair in a tight eigenvalue cluster [4].

Newton's method also requires the calculation and factorization of the Jacobian matrix at each iteration step, which renders the approach computationally expensive for very large systems. Therefore, in this paper we also examine Iterative Refinement alternatives based on a constant or modified iteration matrix. These reduce significantly the computations per iteration, much as in the fast decoupled power flow compared to a NewtonRaphson power flow. Of course, in any case, the need for good initial values still remains.

Consequently, the approach we describe in this paper consists of two stages. Stage 1 is a traditional, robust start-up process. We chose Subspace Iterations for this purpose. Stage 2 is the Iterative Refinement of the solution obtained in Stage 1. There are two ways in performing these calculations:

- Block approach. When the dimension of the subspace is specified in advance, it can be calculated as a whole.

- Sequential approach. The subspace can be built up gradually by adding a new eigenpair to the already calculated set.

The sequential approach is clearly superior to the block approach because of its flexibility. However, we shall use the latter, for notational simplicity in the mathematical derivations.

\section{Subspace Iterations}

Subspace Iterations are a generalization of the classical inverse power iteration method for a multi-dimensional subspace [14,16]. The basic algorithm consists of iteratively solving

$$
\left(A^{\prime}-\sigma l\right) X^{r}=Q^{r-1}
$$

where $\sigma$ is a shift point, chosen as the center of the eigenvalue cluster we intend to identify; $Q^{r-1}$ is a unitary matrix of columns representing an orthonormal basis of the subspace at the end of step $r-1 ; X^{r}$ is an updated basis for the same subspace at the end of step $r$. Matrix $X^{r}$ is subsequently orthonormalized by the Gram-Schmidt algorithm. This amounts to the $\mathrm{QR}$ decomposition

$$
X^{r}=Q^{r} R^{r}
$$

The algorithm starts with $Q^{0}$ being the first columns of the identity matrix. The asymptotic rate of convergence is determined by the distance to the shift point of the closest eigenvalue outside the cluster [16]. The process is robust even though it may require many iterations to attain a satisfactory level of accuracy.

\section{Iterative Refinement}

We review Newton's method for a single eigenpair [4] in order to facilitate the generalization of the method for the case of eigenvalue clusters. For this purpose we write (6) in the form

$$
f \equiv A^{\prime} x-x \lambda=0
$$

We note that the eigenvector $x$ is normalized by setting one of its nonzero elements to a constant, for example unity. In this way, the number of unknowns (including $\lambda$ ) is equal to the number of equations. Thus the problem is well-posed and, consequently, its truncated Taylor expansion leads, in general, to the well-conditioned linear problem

$$
f_{o}+\left(A^{\prime}-\lambda I\right) \Delta x-x \Delta \lambda=0
$$

where $f_{0}$ is the current mismatch given by (10). In the case of a cluster of $m$ eigenvalues, problem (10) becomes

$$
F \equiv A^{\prime} X-X \Lambda=0
$$

where $\Lambda$ is the diagonal matrix of the $m$ eigenvalues $\lambda_{k}$, and $X$ the matrix of the corresponding eigenvectors $x_{k}$, for $k=1,2, \ldots, m$. Since matrix $A^{\prime}$ is generally not defective, the $m$ eigenvectors are linearly independent, spanning an $m$-dimensional subspace.

Consider an $m \times m$ matrix $M$ similar to $\Lambda$, i.e.,

$$
M=Z \wedge Z^{-1}
$$

Substituting $\Lambda$ from (13) into (12) yields

$$
F \equiv A^{\prime} X-X M=\mathrm{O}
$$

where $X$ is now a matrix with columns (generally not eigenvectors) spanning the same subspace. Equation (14) is the invariant subspace generalization of the conventional formulation (12) of the eigenvalue-eigenvector problem. We use the invariant subspace formulation because of the resultant computational advantages that become apparent in the following.

The truncated Taylor expansion of (14) is

$$
F_{o}+A^{\prime} \Delta X-\Delta X M-X \Delta M=0
$$

where $F_{o}$ is the current mismatch given by (14)

Equation (15) is used to iteratively update $X$ and $M$ until (14) is satisfied. For this purpose, in a departure from Newton's method in order to facilitate the derivations, we make the approximation

$$
M=\sigma I
$$

This is based on the spectral decomposition (13) of $M$ where we note that $\Lambda \simeq \sigma I$, since the eigenvalues lie in a close neighborhood of the shift point $\sigma$. Thus (15) becomes

$$
F_{o}+\left(A^{\prime}-\sigma I\right) \Delta X-X \Delta M=0
$$

which is analogous to (11). Since in (17) $\Delta M$ consists of $\mathrm{m}^{2}$ unknowns, an equal number of unknowns must be removed from $\Delta X$ 
so that the total number of unknowns equals the number of equations. We achieve this by normalizing the $m \times m$ upper block of $X$ by setting it to a constant. Subsequently, (17) is rearranged as follows:

$$
B \Delta Y=-F_{0}
$$

where $B$ is obtained from the matrix $A^{\prime}-\sigma l$ of (17) by replacing its first $m$ columns by $-X$; and $\Delta Y$ is composed of $\Delta X$ with the upper $m \times m$ block of zeros replaced by $\Delta M$.

In the simplest form of the Iterative Refinement method, the iteration matrix $B$ in (18) is kept constant. This implies that its first $m$ columns are not modified even though $X$ is updated when solving (18). Of course, for the same reason, the shift point $\sigma$ in $B$ is also kept constant.

To start the Iterative Refinement, matrix $X$ is obtained as the solution of the Subspace Iteration stage. Matrix $M$ is obtained from the Rayleigh quotient [15] given by

$$
M=X^{H} A^{\prime} X
$$

for the orthonormal matrix $X$.

After the Iterative Refinement has converged, the eigenvalues of $A^{\prime}$ in the cluster are obtained as the eigenvalues of $M$, according to (13). Substitution of (13) into (14) yields

$$
A^{\prime}(X Z)-(X Z) \Lambda=0
$$

which is similar to (12). Thus the eigenvectors of $A^{\prime}$, associated with the eigenvalues $\Lambda$ in the cluster, are given as the columns of the matrix $X Z$

\section{Algorithmic Alternatives}

\section{Sequential Approach}

In the previous sections we have presented a methodology for identifying the eigenvalues in a cluster of specified size and the associated eigenvectors, in a single, simultaneous, block computation. Often, however, the size of a cluster is not determined in advance. Therefore, it is desirable to identify a cluster sequentially by augmenting the already calculated set of eigenpairs, one at a time. This procedure is straightforward for the Subspace Iteration method. For the Iterative Refinement it is described in the following.

By Schur's theorem $[14,16]$, any matrix $M$ is unitarily similar to an upper triangular matrix. Therefore, based on such a transformation, we shall consider now that matrix $M$ in (14) is upper triangular. This implies that the normalization of $X$, discussed previously, will consist in setting to a constant only the upper triangular part of $X$. Thus the total number of unknowns, including those in the triangular matrix $M$, equals the number of equations. In the sequential approach, in equation (17) only the last columns of $F_{o}$, $\Delta X$ and $\Delta M$ are nonzero, since the already calculated eigenpairs are assumed to have fully converged. Consequently, we extract the last column from (17), as follows:

$$
f_{o}+\left(A^{\prime}-\sigma I\right) \Delta x-X \Delta r=0
$$

Here, $\Delta r$ represents the last column of the upper triangular matrix $\Delta M$. Similarly, (18) gives

$$
B \Delta y=-f_{o}
$$

The dimension of $\Delta r$ in (21) is $k$, when eigenpair $k$ is being identified. Correspondingly, $X$ in (21) has $k$ columns. Since $k=1,2, \ldots$, the dimension of $X$ increases as the sequential process advances. Recall that $B$ in (22) has $-X$ for its first $k$ columns. Therefore, $B$ has to be continually modified. Implementation details necessary to assure the efficiency of the described procedures will be discussed in a later section.

At the end of the refinement process, the converged new vector is orthonormalized to the previously calculated set of vectors. This induces a modification only in the last column of matrix $M$.

\section{Quasi-Newlon Approach}

The constant-matrix sequential Iterative Refinement described in the previous section can be modified by updating the iteration matrix $B$ of (22) at each step, both in terms of the matrix $X$ that forms its first columns and the shift point $\sigma$. At this point we do not suggest a complete refactorization of matrix $B$ that would lead to a true Newton algorithm. Instead, we now update accurately only the part of $B$ that corresponds to $X$. This is done with little computational effort, as shown later.

The effect of modifying the shift point $\sigma$ is obtained without a refactorization by taking advantage of the fact that the required correction $\varepsilon$ of the shift is small compared to the shift $\sigma$ itself. This is so because $\varepsilon$ cannot be larger than the radius of the cluster, which we assume to be small. The details of this update are shown in Appendix A.

The iteration matrix $B$ of (22), updated as suggested here, is very close, yet not identical, to the true Jacobian matrix. Therefore, the convergence of the Quasi-Newton Iterative Refinement (22) is still linear, albeit faster, as demonstrated in the results shown later. I is fastest for the first identified eigenvalues that are the closest to the shift point $\sigma$.

\section{Newton's Method}

The changes in the iteration matrix of the Quasi-Newton approach, described above, resulted in a good approximation of the Jacobian matrix. At the additional cost of complete matrix refactorizations, a true Newton method has also been implemented.

We emphasize that this invariant subspace implementation of Newton's method is immune to problems that may arise from the closeness of eigenvalues. These problems are the difficulty of identifying all eigenvalues in a tight cluster and slow convergence. In fact, in the case of multiple eigenvalues, it is known that Newton's method is only linearly convergent [19].

In all instances, the fast quadratic convergence achieved has more than offset the additional computational cost of sparse refactorizations.

\section{Algorithm}

In this section we shall discuss several aspects related to the efficient implementation of the basic ideas outlined above.

\section{Sparse Implementation}

Since the problem at hand consists of both differential and algebraic equations (4) leading to (5), rather than the reduced forms (10) and (12), the Subspace Iteration process $(8)$ becomes

$$
\left[\begin{array}{cc}
A-\sigma I & B \\
C & D
\end{array}\right]\left[\begin{array}{c}
X^{r} \\
U^{r}
\end{array}\right]=\left[\begin{array}{c}
Q^{r-1} \\
O
\end{array}\right]
$$

The advantage of this formulation is that the coefficient matrix in (23) is sparse. The solution now contains also $U^{r}$, corresponding to the algebraic variables. The orthogonalization (9) of $X^{r}$ will induce an update of $U^{r}$ (needed in the Iterative Refinement) via the second equation in (23). This operation can be very efficiently performed as a simple extension of the Gram-Schmidt algorithm.

Similarly, in the Iterative Refinement, instead of (14) we use

$$
\left[\begin{array}{l}
F \\
G
\end{array}\right] \equiv\left[\begin{array}{ll}
A & B \\
C & D
\end{array}\right]\left[\begin{array}{l}
X \\
U
\end{array}\right]-\left[\begin{array}{l}
X \\
\mathrm{O}
\end{array}\right] M=\mathrm{O}
$$

Accordingly, (17) becomes

$$
\left[\begin{array}{l}
F_{o} \\
G_{o}
\end{array}\right]+\left[\begin{array}{cc}
A-\sigma I & B \\
C & D
\end{array}\right]\left[\begin{array}{c}
\Delta X \\
\Delta U
\end{array}\right]-\left[\begin{array}{l}
X \\
\mathrm{O}
\end{array}\right] \Delta M=\mathrm{O}
$$

which can be rearranged in a form similar to (18) with a sparse coefficient matrix. It will yield updates for $M, X$, and $U$.

In the sequential approach, (23) and (25) are used only for the new vector, the last basis vector that augments the subspace. 


\section{Efficiency Measures}

In the Subspace Iteration, since all previous vectors are already orthonormal, only the new vector has to be orthogonalized with respect to the existing ones. This operation requires significantly less computation than the orthonormalization (9) used in the block approach.

At the Iterative Refinement stage, since the column dimension of $X$ increases as the subspace is augmented, there is a need for modifications in the coefficient matrix $B$ of (18) or (22). This modification affects a single column at each augmentation. Therefore, it is of rank one and could be implemented using the Matrix Modification Lemma [20]. However, we follow the more direct approach shown in Appendix B.

\section{Adaptive Strategy}

As already emphasized, the Iterative Refinement (IR) methods, which (for instance in the case of Newton's method) could be very fast, are crucially dependent on good initial values. These are obtained by a Subspace Iteration (SI) method, which may be slower, but is robust. Since it is our interest to start with SI but switch to IR as soon as we obtain a solution that is sufficiently good for IR to converge, we implemented an adaptive strategy that renders the overall algorithm both fast and robust. According to this strategy, when SI satisfies a first convergence tolerance, the algorithm switches to IR. The IR iterations are monitored so that if the convergence is very slow, or if the process diverges, the algorithm reverts from IR to SI, which is continued from its previous solution until a more accurate solution is obtained. Then the $\mathbb{R}$ process is tried again. This automated strategy assures a good overall performance of the algorithm.

\section{COMPUTATIONAL RESULTS}

All simulations were performed on an IBM PC compatible computer equipped with an Intel $486 \mathrm{DX} 2-66 \mathrm{MHz}$ processor and $8 \mathrm{MB}$ of RAM. The high-performance numeric computation and visualization software package MATLAB version 4.0 was used for the simulations. The algorithms were implemented as MATLAB scripts (M-files).

\section{Test Systems and Modeling}

For the purpose of testing the performance of the algorithms, we used the IEEE standard test systems of $14,30,57$ and 118 buses [21]. The same dynamic model was used for all machines. It includes two $\mathrm{d}$-axis and two q-axis circuits, and a fast exciter. This model introduces 7 state variables. Typical values were used for the machine constants, according to the individual machine ratings. All static loads were converted to constant admittances after the voltages have been obtained by power flow calculations.

Table 1 lists the characteristics of the test systems used. The number of states (state variables) corresponds to the order of matrices $A$ and $A^{\prime}$. It is the total number of eigenvalues for the system. The number of equations corresponds to the order of the sparse coefficient matrix of (23). The density of this matrix is also shown.

\section{Validation Results}

Calculations in MATLAB are performed in double-precision. However, since the identification of eigenpairs is in all practical cases satisfactory at the single-precision level, a final convergence tolerance of $10^{-6}$ was used in all methods. In the Subspace Iteration (SI) method, the mismatch was the infinite norm (maximum absolute

\begin{tabular}{|c||c|c|c|c|}
\hline \multicolumn{5}{|c|}{ Table 1. Test Systems } \\
\hline \hline System & Machines & States & Equations & Density \\
\hline IEEE-14 & 5 & 35 & 63 & $11.31 \%$ \\
IEEE-30 & 6 & 42 & 102 & $6.96 \%$ \\
IEEE-57 & 7 & 49 & 163 & $4.50 \%$ \\
IEEE-118 & 34 & 238 & 474 & $1.60 \%$ \\
\hline
\end{tabular}

value) of the difference between the current invariant subspace vector(s) and the one(s) at the previous iteration. In the constantmatrix (CM) Iterative Refinement, Quasi-Newton (QN), and Newton (N) methods, the mismatch was the infinite norm of the right-handside matrix $F_{o}$ of (18) (or the right-hand-side vector $f_{o}$ of (22)).

For each system, all eigenpairs were also calculated through a complete eigenanalysis by the $\mathrm{QR}$ algorithm for the purpose of comparison and validation. The various methods tested converged to the same eigenpairs. The block method with specified cluster size of $m$ eigenvalues always converged to the $m$ closest eigenvalues to the shift point used. Similarly, the sequential methods consistently converged to the same eigenvalues in the order of increasing distance from the shift point.

The theory behind the Subspace Iteration (SI) method [16] indicates that the convergence rate of a particular eigenvalue depends on the ratio of the distance of this eigenvalue to the shift point to the distance of the next closest eigenvalue to the shift point. In order to illustrate this fact, we consider the eigenvalue cluster of the 30-bus test system, shown in Table 2 . Table 3 compares the convergence properties for the various sequential algorithms as they obtain the first three eigenvalues of the cluster. The convergence tolerance was set to $10^{-3}$ for the Subspace Iteration (SI) method. SI is subsequently followed by either constant-matrix (CM) Iterative Refinement, or Quasi-Newton (QN), or Newton (N) with convergence tolerance set to $10^{-6}$.

Table 3 lists the mismatch at the last three iterations for each method and each eigenvalue. The last column clearly demonstrates the expected quadratic convergence of Newton's method. If very high accuracy is important, then clearly no other method comes even close to Newton's. The other methods converge linearly. The average rate of convergence (current mismatch versus previous one) is also included in the table for each of these methods and each eigenvalue. As expected, the convergence rate for SI is roughly equal to the corresponding eigenvalue distance ratio, listed in the previous table. $\mathrm{CM}$ has similar convergence properties whereas $\mathrm{QN}$ converges faster.

\begin{tabular}{||c|c|c|c|c||}
\hline \multicolumn{5}{|c|}{ Table 2. Eigenvalue Cluster } \\
\hline \hline Shift Point & No. & Eigenvalue & Distance & Ratio \\
\hline & 1 & $-1.1648+\mathrm{j} 8.2123$ & 0.2687 & 0.5526 \\
$-1.00+\mathrm{j} 8.00$ & 2 & $-1.4863+\mathrm{j} 8.0004$ & 0.4863 & 0.9190 \\
& 3 & $-1.5053+\mathrm{j} 8.1571$ & 0.5292 & 0.9424 \\
& 4 & $-1.3895+\mathrm{j} 8.4044$ & 0.5615 & \\
\hline
\end{tabular}

\begin{tabular}{|c|c|c|c|c|}
\hline \multicolumn{5}{|c|}{ Table 3. Convergence Properties } \\
\hline \multirow{2}{*}{ No. } & \multicolumn{4}{|c|}{ Mismatch/Average Convergence Rate } \\
\hline & SI & $\mathrm{CM}$ & $\mathrm{QN}$ & $\mathbf{N}$ \\
\hline \multirow{4}{*}{1} & $3.216 \times 10^{-2}$ & $2.484 \times 10^{-6}$ & $8.019 \times 10^{-6}$ & $8.675 \times 10^{-4}$ \\
\hline & $1.669 \times 10^{-2}$ & $1.405 \times 10^{-6}$ & $2.489 \times 10^{-6}$ & $1.644 \times 10^{-6}$ \\
\hline & $7.861 \times 10^{-3}$ & $7.893 \times 10^{-7}$ & $7.908 \times 10^{-7}$ & $1.097 \times 10^{-11}$ \\
\hline & 0.57 & 0.588 & 0.380 & \\
\hline \multirow{4}{*}{2} & $1.196 \times 10^{-2}$ & $1.438 \times 10^{-6}$ & $1.651 \times 10^{-6}$ & $9.910 \times 10^{-2}$ \\
\hline & $1.036 \times 10^{-2}$ & $1.124 \times 10^{-6}$ & $1.099 \times 10^{-6}$ & $1.662 \times 10^{-4}$ \\
\hline & $9.014 \times 10^{-3}$ & $8.756 \times 10^{-7}$ & $8.693 \times 10^{-7}$ & $9.376 \times 10^{-8}$ \\
\hline & 0.886 & 0.893 & 0.775 & \\
\hline \multirow{4}{*}{3} & $1.077 \times 10^{-2}$ & $1.047 \times 10^{-6}$ & $1.270 \times 10^{-6}$ & $1.035 \times 10^{-1}$ \\
\hline & $1.018 \times 10^{-2}$ & $1.005 \times 10^{-6}$ & $1.128 \times 10^{-6}$ & $4.803 \times 10^{-5}$ \\
\hline & $9.603 \times 10^{-3}$ & $\underline{9.644 \times 10^{-7}}$ & $9.998 \times 10^{-7}$ & $8.262 \times 10^{-9}$ \\
\hline & 0.977 & 0.961 & 0.894 & \\
\hline
\end{tabular}




\section{Comparative Results}

If we rank the sequential methods in terms of computational cost per iteration, first come SI and CM with roughly the same performance. QN is considerably slower because of the more computationally expensive solution update (refer to Appendices A and B). Finally, Newton's $(N)$ method is the most expensive due to the refactorization of the Jacobian matrix. Obviously, the most efficient combination depends on the system size, the size of the cluster and the capabilities of the available computer hardware and software.

In all algorithmic combinations, the first stage (SI) is slow but robust. Therefore, we developed the adaptive strategy that attempts, in successive cycles, to switch from the first stage to the second as soon as the former satisfies a weak error tolerance. This strategy assures the robustness of the combined process. It is most important for the SI-CM combination since the approximations in the iteration matrix of CM render this method more sensitive to the inaccuracy of the initial solution. In contrast, the strategy was not necessary for Newton's method where even a rough initial solution has always assured convergence in about three iterations. Table 4 illustrates the effect of the adaptive strategy in the SI-CM method combination for a shift point of $-1+j 7$ for the 118 -bus system. The adaptive strategy cycles are shown for two eigenvalues. The mismatch of the last iteration of each method is shown. The initial convergence tolerance in $S I$ is set to $10^{-1}$ and decreases by a factor of 10 for each cycle. Note that the iteration count shown in Table 4 for each method is cumulative.

In order to compare the performance of the sequential algorithms, they were all applied to calculate the same eigenvalue cluster, shown in Table 5. This cluster belongs to the 118 -bus test system. Table 6 lists the adaptive strategy cycles, the first and second stage iterations and the CPU time in seconds for all methods. The results of these tests have clearly shown the superiority of Newton's method over the other alternatives. The SI column corresponds to Subspace Iterations with convergence tolerance of $10^{-6}$.

The MA column corresponds to the Modified Arnoldi (MA) method [11] with 5 guard vectors. We note that this value for the number of guard vectors gave the best results for this case. We also note that this parameter has significant effect on the performance of the method and its best value is not generally known. Modified Amoldi compared unfavorably with the rest of the methods in all our simulations. The reason is that the MA algorithm, which is inherently sequential, operates on the whole invariant subspace, even

\begin{tabular}{|c|c|c|c|c|}
\hline Eigenvalue & Cycle & Method & Iterations & Mismatch \\
\hline \multirow{2}{*}{$-1.180+j 7.1637$} & 1 & $\begin{array}{c}\text { SI } \\
\mathrm{CM}\end{array}$ & $\begin{array}{r}10 \\
5\end{array}$ & $\begin{array}{l}8.256 \times 10^{-2} \\
1.442\end{array}$ \\
\hline & 2 & $\begin{array}{c}\mathrm{SI} \\
\mathrm{CM}\end{array}$ & $\begin{array}{l}17 \\
25 \\
\end{array}$ & $\begin{array}{l}4.763 \times 10^{-3} \\
7.025 \times 10^{-7}\end{array}$ \\
\hline \multirow{3}{*}{$-0.689+j 7.2061$} & 1 & $\begin{array}{c}\mathrm{SI} \\
\mathrm{CM}\end{array}$ & $\begin{array}{r}23 \\
3\end{array}$ & $\begin{array}{l}8.914 \times 10^{-2} \\
1.724\end{array}$ \\
\hline & 2 & $\begin{array}{c}\text { SI } \\
\text { CM }\end{array}$ & $\begin{array}{r}39 \\
7\end{array}$ & $\begin{array}{l}9.100 \times 10^{-3} \\
8.610 \times 10^{-1}\end{array}$ \\
\hline & 3 & $\begin{array}{c}\text { SI } \\
\text { CM }\end{array}$ & $\begin{array}{r}55 \\
216 \\
\end{array}$ & $\begin{array}{l}8.780 \times 10^{-4} \\
9.530 \times 10^{-7}\end{array}$ \\
\hline
\end{tabular}

\begin{tabular}{||c|c|c|c||}
\hline \multicolumn{4}{|c|}{ Table 5. Eigenvalues } \\
\hline \hline Shift Point & No. & Eigenvalue & Distance \\
\hline & 1 & $-0.7667+\mathrm{j} 6.6364$ & 0.2995 \\
$-0.5000+\mathrm{j} 6.5000$ & 2 & $-0.0899+\mathrm{j} 6.2332$ & 0.4892 \\
& 3 & $-0.5184+\mathrm{j} 5.9515$ & 0.5488 \\
& 4 & $-0.2290+\mathrm{j} 7.1193$ & 0.6759 \\
\hline
\end{tabular}

\begin{tabular}{||l||l|r|r|r|r|r||}
\hline \multicolumn{5}{|c|}{ Table 6. Algorithm Performance } \\
\hline \hline \multirow{2}{*}{ No. } & Performance & \multicolumn{5}{c||}{ Method } \\
\cline { 3 - 7 } & & SI & SI-CM & SI-QN & SI-N & MA \\
\hline \hline \multirow{3}{*}{1} & Cycles & & 1 & 1 & 1 & \\
& Stage 1 & \multirow{2}{*}{27} & 5 & 5 & 5 & 3 \\
& Stage 2 & & 19 & 10 & 3 & \\
\hline \multirow{3}{*}{2} & Cycles & & 1 & 1 & 1 & \\
& Stage 1 & \multirow{4}{*}{119} & 19 & 19 & 19 & 3 \\
& Stage 2 & & 108 & 47 & 3 & \\
\hline \multirow{3}{*}{3} & Cycles & & 1 & 1 & 1 & \\
& Stage 1 & \multirow{3}{*}{75} & 20 & 20 & 20 & 3 \\
& Stage 2 & & 52 & 26 & 3 & \\
\hline \multirow{3}{*}{4} & Cycles & & 3 & 2 & 1 & \\
& Stage 1 & \multirow{2}{*}{166} & 77 & 48 & 20 & 5 \\
& Stage 2 & & 162 & 84 & 5 & \\
\hline \hline CPU Time [s] & 142 & 161 & 178 & 62 & 295 \\
\hline
\end{tabular}

though the previous subspace has converged. On the other hand, our algorithms were carefully implemented to operate only on the new vector that augments the invariant subspace.

\section{CONCLUSIONS}

The paper has described a new methodology for the calculation of critical eigenpairs in the small signal stability analysis of power systems. It has the following essential features:

- It deals with clusters of eigenvalues and their associated eigenvectors. The size of the cluster can be defined from the outset or the cluster can be built up gradually. In the latter case, the calculations are restricted only to the new vector that augments the invariant subspace.

- The problem is solved in two stages. The first stage is a Subspace (simultaneous) Iteration to produce good starting values. The second stage is the Iterative Refinement of the solution.

- The Iterative Refinement has several alternatives: a constantmatrix, a Quasi-Newton, and a true Newton approach. The Newton approach, due to its extremely fast, quadratic convergence, is by far the most efficient for problems where high accuracy is essential.

- Only a single, sparse, matrix factorization, except for Newton's method, is needed for the whole process. All the necessary calculations are efficiently performed with little computational effort.

- Many dynamic systems (engineering, biological, economic, etc.) have a structure similar to that of the power system, assumed for this study: dynamic components interconnected by a static network. Therefore, the methodology described could potentially be applied also to other problems of dynamic analysis.

\section{ACKNOWLEDGEMENTS}

The authors wish to thank Professor M. R. Iravani of the University of Toronto and Dr. Lei Wang of Ontario Hydro, for instructive discussions and useful advice. Financial support by the National Sciences and Engineering Research Council of Canada is gratefully acknowledged.

\section{REFERENCES}

[1] R. T. Byerly, R. J. Bennon, and D. E. Sherma, "Eigenvalue Analysis of Synchronizing Power Flow Oscillations in Large Electric Power Synchronizing Power Flow Oscillations in Large Electric Powe Systems", IEEE Transactions on Power Apparatur
Vol. PAS-101, No. 1, January 1982, pp. 235-243.

[2] D. Y. Wong, G. J. Rogers, B. Porretta, and P. Kundur, "Eigenvalue Analysis of Very Large Power Systems", IEEE Transactions on Powe Analysis of Very Large Power Systems",
Systems, Vol. PWRS-3, No. 2, May 1988, pp. 472-480. 
432

[3] N. Martins, "Efficient Eigenvalue and Frequency Response Methods Applied to Power System Small-Signal Stability Studies", IEEE Transactions on Power Systems, Vol. PWRS - 1, No. 1, February 1986 pp. 217-226.

[4] A. Semlyen and L. Wang, "Sequential Computation of the Complete Eigensystem for the Study Zone in Small Signal Stability Analysis of Large Power Systems", IEEE Transactions on Power Systems. Large Power Systems", IEEE Transactions
Vol. PWRS-3, No. 2, May 1988, pp. 715-725.

[5] P. Kundur, G. J. Rogers, D. Y. Wong, L. Wang, and M. G. Lauby, “A Comprehensive Computer Program Package for Small Signal Stability Analysis of Power Systems", IEEE Transactions on Power Systems, Vol. PWRS-5, No. 4, November 1990, pp. 1076-1083.

[6] N. Müller and V. H. Quintana. "A Sparse Eigenvalue-Based Approach for Partitioning Power Network", IEEE Transactions on Power Systems, Vol. PWRS - 7, No. 2, May 1992, pp. 520-527.

[7] I. J. Pérez-Arriaga, G. C. Verghese, and F. C. Schweppe, "Selective Modal Analysis with Applications to Electric Power Systems, Part I: Heuristic Introduction", IEEE Transactions on Power Apparatus
Systems, Vol. PAS-101, No. 9, September 1982, pp. $3117-3125$.

[8] G. C. Verghese, I. J. Pérez-Arriaga, and F. C. Schweppe, "Selective Modal Analysis with Applications to Electric Power Systems, Part II The Dynamic Stability Problem", IEEE Transactions on Power Apparatus and Systems, Vol. PAS-101, No. 9, September 1982, pp. $3126-3134$.

[9] J. L. Sancha and I. J. Pérez-A rriaga, "Selective Modal Analysis of Power System Oscillatory Instability", IEEE Transactions on Power Systems, Vol. PWRS - 3, No. 2, May 1988, pp. 429-438.

[10] N. Uchida and T. Nagao, "A New Eigen-Analysis Mcthod of SteadyState Stability Studies for Large Power Systems: S Matrix Method" IEEE Transactions on Power Systems, Vol. PWRS -3, No. 2, May 1988, pp. 706-714.

[11] L. Wang and A. Semlyen, “Application of Sparse Eigenvalue Techniques to the Small Signal Stability Analysis of Large Powe Systems", IEEE Transactions

[12] D. J. Stadnicki and J. E. Van Ness, "Invariant Subspace Method for Eigenvalue Computation", IEEE Transactions on Power Systems
Vol. PWRS-8, No. 2, May 1993, pp. 572-580.

[13] A. Jennings, Matrix Computations for Engineers and Scientists, John Wiley \& Sons; New York, 1977

[14] G. H. Golub and C. F. Van Loan, Matrix Computations, 2nd ed., John Hopkins University Press, Baltimore, 1989.

[15] G. W. Stewart, Introduction to Matrix Computations, Academic Press, New York, 1973.

[16] D.S. Watkins, Fundamentals of Matrix Computations, John Wiley \& Sons, New York, 1991

[17] Y.Saad, Numerical Methods for Large Eigenvalue Problems: Theory and Algorithms, John Wiley \& Sons, New York, 1992.

[18] G. Gross and A. R. Bergen, “A Class of New Multistep Integration Algorithms for the Computation of Power System Dynamical Response", IEEE Transactions on Power Apparatus and Systems. Vol. PAS-96, No. 1, January/February 1977, pp. 293-306.

[19] G. Dahlquist and A. Björck, "Section 6.7: Multiple Roots", in Numerical Methods, Prentice-Hall, Englewood Cliffs, New Jersey, 1974, p. 242

[20] H. V. Henderson and S. R. Searle, "On Deriving the Inverse of a Sum of Matrices", SIAM Review, Vol. 23, No. 1, January 1981, pp. 53-60.

[21] Y.Wallach, "Section 1.6: Example Networks and Notes" in Calculations and Programs for Power System Networks, Prentice-Hall, Englewood Cliffs, New Jersey, 1986.

\section{APPENDICES}

Appendix A. Solution Update for Small Diagonal Modification

This Appendix describes the technique used for implementing the progressive updates in the iteration matrix $B$ in (22) in each iteration of the Quasi-Newton approach as the shift point $\sigma$ is changed. Let us consider the following system of linear equations:

$$
A x=b
$$

The solution $x$ is given by

$$
x=A^{-1} b
$$

where $A^{-1}$ is a matrix operator denoting efficient operations on $b$ (ordering, forward and backward substitution, etc.). Let us now consider the modified system

$$
(A+\varepsilon I) x^{\prime}=b
$$

where $\varepsilon$ is a small scalar. Premultiplying (A.3) by $A^{-1}$ yields

$$
A^{-1}(A+\varepsilon l) x^{\prime}=A^{-1} b
$$

or

$$
\left(I+\varepsilon A^{-1}\right) x^{\prime}=x
$$

We now use the series expansion

$$
\left(I+\varepsilon A^{-1}\right)^{-1}=I-\varepsilon A^{-1}+\left(\varepsilon A^{-1}\right)^{2}-\cdots
$$

Consequently, the modified solution $x^{\prime}$ can be obtained approximately as follows:

$$
x^{\prime}=\left(I+\varepsilon A^{-1}\right)^{-1} x=\left(I-\varepsilon A^{-1}\right) x=x-\varepsilon A^{-1} x
$$

The approximation in (A.7) is good since $\varepsilon$ is small and $A$ wellconditioned. If, in any case, more accuracy is needed, an additional term in the series expansion of (A.6) can be easily included at a small computational cost.

In the actual Quasi-Newton problem, instead of the full rank identity matrix $I$ in (A.3), we have a matrix with unities only in part of the main diagonal. This somewhat simplifies the operations described above.

Appendix B. Solution Update for Column Modifications

This Appendix demonstrates the technique used for implementing the progressive updates of the iteration matrix $B$ in (22) with every augmentation of the invariant subspace. We start with equations (A.1) and (A.2). Let us now consider a low rank modification on the coefficient matrix of (A.1)

$$
(A+B) x^{\prime}=b
$$

where $B$ is a matrix of zeros, except for the first $k$ nonzero columns that constitute the modifications of the corresponding columns in $A$. Premultiplying (B.1) by $A^{-1}$ yields

$$
A^{-1}(A+B) x^{\prime}=A^{-1} b
$$

or

$$
(I+E) x^{\prime}=x
$$

where $E$ is the result of the operator $A^{-1}$ acting on the columns of $B$. Since only the first $k$ columns of $E$ are nonzero, (B.3) can be written as

$$
\left[\begin{array}{ll}
C_{1} & 0 \\
C_{2} & I
\end{array}\right]\left[\begin{array}{l}
x_{1}^{\prime} \\
x_{2}^{\prime}
\end{array}\right]=\left[\begin{array}{l}
x_{1} \\
x_{2}
\end{array}\right]
$$

The modified solution $x^{\prime}$ can now be obtained by first solving the $k$ th-order square system

$$
C_{1} x_{1}{ }^{\prime}=x_{1}
$$

for $x_{1}{ }^{\prime}$ and then obtaining $x_{2}^{\prime}$ from

$$
x_{2}^{\prime}=x_{2}-C_{2} x_{1}^{\prime}
$$

These operations require very little computational effort since $k$ is small. As an additional efficiency measure in the sequential constant-matrix Iterative Refinement algorithm, the nonzero columns of $E$ are stored as calculated so that the operation $A^{-1} B$ is performed only on the new nonzero column of $B$.

\section{BIOGRAPHIES}

George Angelidis (Member, IEEE) was bom in Athens, Greece, in 1962. He received the Ptychion degree (B.A.Sc) from the Aristotle University of Thessaloniki in 1984, and the M.A.Sc. and Ph.D. degrees from the University of Toronto in 1988 and 1992, respectively, all in Electrical Engineering. He is currently working as a Postdoctoral Fellow in the Department of Electrical and Computer Engineering at the University of Toronto. His research interests are in computer applications in large-scale electric power systems.

Adam Semlyen (Fellow, IEEE) was bom and educated in Rumania where he obtained a Dipl. Ing. degree and his Ph.D. He started his career with an electric power utility and held academic positions at the Polytechnic Institute of Timisoara, Rumania. In 1969 he joined the University of Toronto where he is a professor in the Department of Electrical and Computer Engineering, emeritus since 1988. His research interests include the steady state and dynamic analysis of power systems, electromagnetic transients, and power system optimization. 